\title{
BMJ open Inhaled hypertonic saline in adults hospitalised for exacerbation of cystic fibrosis lung disease: a retrospective study
}

\author{
Alejandro A Pezzulo, David A Stoltz, Douglas B Hornick, Lakshmi Durairaj
}

To cite: Pezzulo AA, Stoltz DA, Hornick DB, et al. Inhaled hypertonic saline in adults hospitalised for exacerbation of cystic fibrosis lung disease: a retrospective e000407. doi:10.1136/ bmjopen-2011-000407

- Prepublication history for this paper is available online. To view these files please visit the journal online (http:// dx.doi.org/10.1136/ bmjopen-2011-000407).

Received 22 September 2011 Accepted 12 March 2012

This final article is available for use under the terms of the Creative Commons Attribution Non-Commercial 2.0 Licence; see http://bmjopen.bmj.com study. BMJ Open 2012;2:

\section{ABSTRACT}

Background: Inhaled hypertonic saline (HTS) improves quality of life and reduces pulmonary exacerbations when given long term in patients with cystic fibrosis (CF). While increasingly being offered for acute pulmonary exacerbations, little is known about the efficacy in this setting.

Objectives: The authors examined the tolerability and efficacy of HTS use among adult subjects hospitalised with a CF pulmonary exacerbation and hypothesised that use of HTS would improve pulmonary function during the admission.

Design: Pilot retrospective non-randomised study. Setting: Single tertiary care centre.

Participants: 45 subjects admitted to the inpatient service for acute CF pulmonary exacerbation in 2006-2007. A subset of 18 subjects who were also admitted in 2005 when HTS was not available was included in the comparative study.

Primary outcome: Change in forced expiratory volume in one second from admission to discharge.

Secondary outcomes: Change in weight from admission to discharge and time to next exacerbation.

Results: Mean age was 32.5 years, and mean length of stay was 11.5 days. HTS was offered to 33 subjects and was well tolerated for a total use of 336 days out of 364 days of hospital stay. Baseline demographics, lung function and sputum culture results were comparable in first and second visits. Use of HTS was not associated with an improvement in forced expiratory volume in one second $(p=0.1)$, weight gain $(p=0.24)$ or in the time to next admission $(p=0.08)$.

Conclusions: These pilot data suggest that HTS is well tolerated during CF pulmonary exacerbation but offers no clear outcome benefits. It is possible that HTS may not have much advantage above and beyond intensive rehabilitation and intravenous antibiotics and may add to hospital costs and treatment burden.

Department of Internal Medicine, University of lowa Carver College of Medicine, Iowa City, Iowa, USA

Correspondence to Dr Lakshmi Durairaj; lakshmi-durairaj@uiowa.edu

\section{BACKGROUND}

Lung disease is the leading cause of morbidity and mortality in patients with cystic fibrosis (CF). It is characterised by

\section{ARTICLE SUMMARY}

\section{Article focus}

- Long-term use of inhaled HTS improves pulmonary function and decreases exacerbation frequency in patients with CF.

- Despite lack of available data regarding efficacy and safety, inhaled HTS is frequently used during hospitalisation for exacerbation of CF pulmonary disease.

\section{Key messages}

- This retrospective non-randomised study suggests that inhaled HTS is well tolerated during hospitalisation for exacerbation of CF pulmonary disease.

- Short-term (14 days) use of inhaled HTS provided no added improvement over standard care (intravenous antibiotics and chest physical therapy) in pulmonary function, weight gain or time to next hospitalisation.

Strengths and limitations of this study

- This study allowed within-patient comparison of standard treatment with or without inhaled HTS in a relatively homogeneous adult population in an inpatient setting.

- The main limitation of this study is the retrospective and non-randomised design and unavailability of detailed compliance and side effect data. Sample size was adequate for the objectives of the study.

chronic bacterial colonisation and recurrent infections of the airways, which have been evident manifestations of the disease since the term 'cystic fibrosis' was first coined. ${ }^{12}$ Mutations in the CF transmembrane conductance regulator gene result in defective epithelial chloride transport in human airway epithelia, but the downstream consequences of this defect and how they result in $\mathrm{CF}$ lung disease remain to be further elucidated. ${ }^{3}{ }^{4}$ The clinical course of patients with $\mathrm{CF}$ is characterised by periods of 'stable' disease, interspersed by recurrent 
exacerbations during which symptoms such as sputum production and cough tend to acutely increase in intensity and are associated with increased bacterial density in the lungs and hypoxaemia. ${ }^{5-8}$

During acute CF lung disease exacerbation, usual treatment involves intravenous antibiotics, enhanced nutritional supplementation and airway clearance therapies (eg, frequent chest physiotherapy, inhaled albuterol, aerobic exercise) ${ }^{79}$ Inhaled rhDNase can improve pulmonary function when used long term, albeit at a relatively high cost. ${ }^{10-12}$ Inhaled hypertonic saline (HTS) is an attractive addition because it is inexpensive, fairly well tolerated and acutely increases whole-lung mucus clearance. Long-term treatment with inhaled HTS has been shown to improve pulmonary function and decrease exacerbation frequency. ${ }^{13-18}$ However, HTS use during an exacerbation may be potentially more harmful by causing more bronchospasm or haemoptysis. To our knowledge, no studies have examined the safety profile and efficacy of inhaled HTS treatment given during CF pulmonary exacerbation.

In this pilot retrospective study, we examine the impact of HTS use among a cohort of adult subjects hospitalised with $\mathrm{CF}$ pulmonary exacerbation compared with the same group of subjects during a previous exacerbation when HTS was not used. We hypothesised that use of inhaled HTS in addition to standard care during hospitalisation for acute exacerbation of $\mathrm{CF}$ would result in greater improvement in common end points such as pulmonary function, body weight and the time to next exacerbation when compared with standard of care.

\section{METHODS}

The study was approved by the University of Iowa Institutional Review Board (200710793). This is a retrospective chart review of all adult admissions for CF lung disease exacerbation at the University of Iowa Hospitals and Clinics during the years 2006 and 2007 when inhaled HTS was first offered to patients. Standard care consists of intravenous antibiotics chosen based on the most recent sputum culture and susceptibility testing, nutrition supplementation, pulmonary rehabilitation and intensive mucus clearance techniques such as chest physical therapy four times per day. Study subjects had a diagnosis of $\mathrm{CF}$ based on a sweat chloride test or a nasal voltage test, and genotyping of the CFTR gene. For the comparative study, data were abstracted from the admission during which HTS ('second visit') was first used and compared with an earlier admission in the previous year (2005) in which HTS was not used ('first visit').

Variables studied include age and gender, body mass index on admission and discharge, duration of hospitalisation, intravenous antibiotics use, CF mutation, use of chest physical therapy, sputum culture results, time to next exacerbation and HTS use during subsequent hospitalisations. Baseline pulmonary function values were obtained from testing performed on admission day or in the previous 7 days. During admission, pulmonary function testing was performed twice a week. One subject was discharged after 2 days with a peripherally inserted central catheter to complete antibiotics at home. Subjects received an inhaled $\beta_{2}$ agonist to minimise the risk of bronchospasm followed by a $4 \mathrm{ml}$ dose of $7 \%$ HTS solution twice daily through a Pari LC nebuliser (PARI Respiratory Equipment, Midlothian, VA, USA). Compliance data are reported as total days of HTS use per total hospitalisation days in the study population. For the comparison study, only subjects who used HTS at least 7 days were included. Main outcome variable of comparison was change in per cent of predicted forced expiratory volume in one second $\left(\mathrm{FEV}_{1}\right)$ from baseline to discharge. Subjects who failed to reach $90 \%$ of their best per cent of predicted $\mathrm{FEV}_{1}$ in the 6 months previous to hospitalisation were defined as non-responders to therapy. ${ }^{19}$ Other outcomes analysed include length of stay, compliance, time to next admission and weight gain.

\section{Power analysis}

Power analysis suggested a paired sample size of 18 patients to detect a change of $10.9 \%$ in $\mathrm{FEV}_{1}$ (primary outcome) or weight with a conservative between-group SD of $16 \%$, with $80 \%$ power at two-tailed $\alpha$ of 0.05 . Observed power analysis for primary and secondary outcomes is detailed in the Discussion section.

\section{Outcomes analysis}

Data were analysed using Prism 5 for Mac OS (Graphpad Software) and SAS V.9.2 (SAS Institute). Power analysis was performed using Statmate (Graphpad Software). Univariate data are reported as means and SD and proportions. Paired t test was used for comparisons using a 5\% level of significance. Mixed models for repeated measures were used to compare $\mathrm{FEV}_{1}$ change from baseline in the comparison study. Fisher's exact test was used to compute $p$ value of contingency analysis of 'nonresponders' and 'responders' in both visits and to analyse categorical data in table 1.

\section{RESULTS}

\section{Study participants}

Between January 2006 and December 2007, 45 subjects were admitted to the CF inpatient service at the University of Iowa Hospitals and Clinics. Mean age at admission for this subject population was $32.5 \pm 10.1$ years, and mean length of stay was $11.1 \pm 4.1$ days $(\mathrm{p}>0.05$, unpaired $\mathrm{t}$ test). Forty-six per cent of these subjects were men. Of the 45 subjects, 25 had an admission in the previous year. All 25 subjects had pulmonary function testing performed around time of admission and at discharge. Of these 25 subjects, 18 had no history of asthma or recent haemoptysis and thus inhaled HTS was offered. These 18 subjects were therefore included in the comparative study as described in the Methods section. Baseline characteristics at admission for subjects who fulfilled inclusion criteria into the comparative study are 
Table 1 Patient characteristics during admission*

\begin{tabular}{|c|c|c|}
\hline Characteristic & First visit & Second visit \\
\hline Age (years), mean $\pm S D$ & $33.6 \pm 9.5$ & $35.0 \pm 9.3$ \\
\hline Male, $\%(n)$ & $77(14)$ & $77(14)$ \\
\hline Body mass index $\left(\mathrm{kg} / \mathrm{m}^{2}\right)$, mean $\pm \mathrm{SD}$ & $20.8 \pm 3.2$ & $21.7 \pm 3.5$ \\
\hline $\mathrm{FEV}_{1}$ (per cent of predicted), mean \pm SD & $39.8 \pm 13.9$ & $44.2 \pm 16.4$ \\
\hline FVC $(I)$, mean \pm SD & $2.7 \pm 1.1$ & $3.2 \pm 1.2$ \\
\hline$\Delta \mathrm{F} 508$ homozygous, \% (n) & $61(11)$ & $61(11)$ \\
\hline Inhaled tobramycin use, \% (n) & $11(2)$ & $16.6(3)$ \\
\hline Inhaled HTS use at home, $\%(n)$ & $0(0)$ & $5(1)$ \\
\hline Inhaled rhDNase use at home, \% (n) & $50(9)$ & $33(6)$ \\
\hline Pancreatic insufficiency, \% (n) & $83(15)$ & $83(15)$ \\
\hline Diabetes mellitus, \% (n) & $39(7)$ & $39(7)$ \\
\hline Pseudomonas aeruginosa in sputum, \% (n) & $72(13)$ & $83(15)$ \\
\hline Sputum density of $P$ aeruginosa (log CFU), mean \pm SD & $7.1 \pm 7.0$ & $7.0 \pm 6.9$ \\
\hline Staphylococcus aureus, \% (n) & $33(6)$ & $39(7)$ \\
\hline Length of stay $(d)$, mean $\pm S D$ & $13.0 \pm 3.9$ & $12.0 \pm 4.5$ \\
\hline
\end{tabular}

shown in table 1. During the entire study period, use of HTS at home prior to admission was uncommon $(0 \%$ of subjects during the first visit and $5 \%$ during the second visit). The proportion of patients using inhaled tobramycin or rhDNase was similar prior to both admission ( $p>0.05)$, and the prevalence of colonisation with Pseudomonas aeruginosa or Staphylococcus aureus was also similar in both admissions $(\mathrm{p}>0.05)$.

HTS is well tolerated by the majority of subjects hospitalised for acute exacerbation of CF

During the study period, inhaled HTS was offered to 34 out of 45 subjects, of whom 33 used it for at least 1 day. The remaining 11 subjects were not offered inhaled HTS due to history of asthma or recent haemoptysis. Among 33 subjects, there was a collective use of HTS for a total of 336 days out of 364 days of hospitalisation. Of the 28 total discontinuation days, 24 were explained by side effects (wheezing and mild haemoptysis) in two subjects. Twenty-seven subjects used HTS for more than 7 days. These data suggest that inhaled HTS is well tolerated by subjects hospitalised for exacerbation of $\mathrm{CF}$ lung disease.

HTS does not improve pulmonary function during hospitalisation for acute exacerbation of CF

Among the 18 subjects included in the comparative study, average change in percentage predicted $\mathrm{FEV}_{1}$ from baseline to discharge (in percentage points) was $12.2 \%(95 \%$ CI $7.7 \%$ to $16.5 \%)$ when HTS was not used and $8.9 \%$ (95\% CI $4.9 \%$ to $12.9 \%$ ) when HTS was used (figure 1A), suggesting that inhaled HTS resulted in decreased improvement in $\mathrm{FEV}_{1}$ at discharge $(\mathrm{p}=0.02$, $95 \%$ CI $0.5 \%$ to $5.9 \%$ ). However, the mean percentage predicted $\mathrm{FEV}_{1}$ at admission was higher when HTS was used $(p=0.04)$ (figure $1 B)$, while the average discharge $\mathrm{FEV}_{1}$ values were not significantly different $(p=0.46)$ between the two visits (figure $1 \mathrm{C}$ ). The data suggest that the decrease in $\mathrm{FEV}_{1}$ improvement when inhaled HTS was used is therefore likely due to a higher admission $\mathrm{FEV}_{1}$ during that hospitalisation. A mixed-model adjustment for the differences in $\mathrm{FEV}_{1}$ at baseline
Figure 1 Forced expiratory volume in one second $\left(\mathrm{FEV}_{1}\right)$. The admission (year 2005) in which hypertonic saline was not used (-) was compared with the admission (year 2006 or 2007) in which hypertonic saline was used (+). (A) Change from admission to discharge $\mathrm{FEV}_{1}$. Units are change in total percentage of predicted. Mean $\pm 95 \% \mathrm{Cl}$ are shown. Per cent predicted $\mathrm{FEV}_{1}$ at admission (B) or discharge (C). Units are percentage of predicted. Lines join data from both visits for the same subject. $n=18$. * $p \leq 0.05 ; N S, p>0.05$.
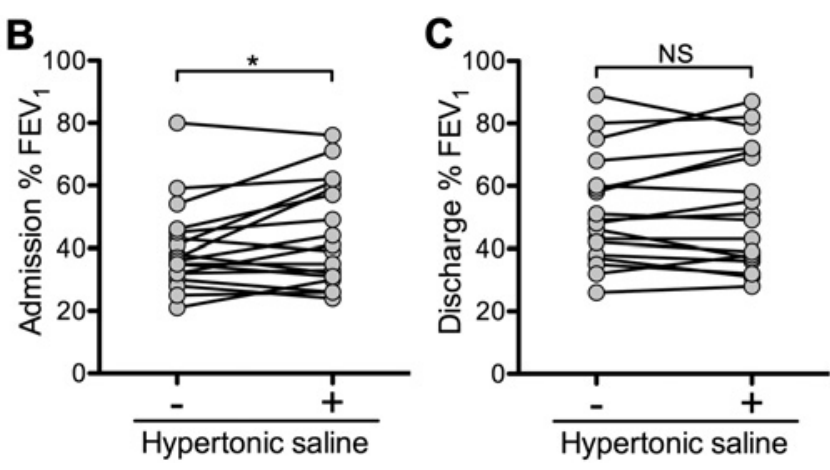
showed no changes in $\mathrm{FEV}_{1}$ improvement when inhaled HTS was used $(p=0.10)$. Next, we analysed the number of subjects who failed to reach at least $90 \%$ of their best per cent $\mathrm{FEV}_{1}$ in the 6 months prior to admission at the end of hospitalisation. This strategy attempts to minimize the confounding effects of week-to week variability in $\mathrm{FEV}_{1} \cdot{ }^{19}$ Four subjects were 'non-responders' when HTS was not used and two when HTS was used $(p=0.65)$. Overall, these data suggest that use of inhaled HTS during hospitalisation for exacerbation of CF lung disease does not result in improved or worsened $\mathrm{FEV}_{1}$ over standard of care in this small population.

HTS does not augment weight gain during hospitalisation for acute exacerbation of CF

In our experience, subjects hospitalised for exacerbation of CF lung disease gain weight during the visit, probably as a result of enhanced caloric intake and/or control of the inflammatory state in the lungs resulting from infection. Figure 2 shows that the addition of inhaled HTS was not associated with an improved or worsened weight gain during hospitalisation. Average weight gain was $3.4 \%$ (95\% CI $0.44 \%$ to $6.3 \%)$ when HTS was not used and $1.5 \%$ (95\% CI $-1.3 \%$ to $4.3 \%$ ) when HTS was used ( $\mathrm{p}=0.24,95 \%$ CI -1.4 to 5.0$)$.

HTS does not increase time to next admission after an acute exacerbation of CF

Since inhaled HTS was used during hospitalisation but not as outpatient in our clinical practice through this study period and in the year 2008, we were able to study the effect of inpatient HTS on time to next admission for pulmonary exacerbation. Average time to next admis-

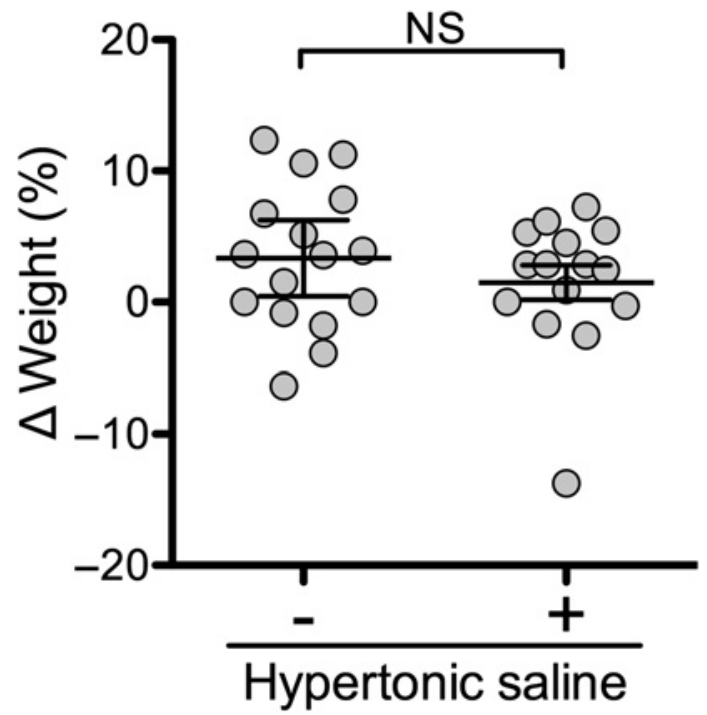

Figure 2 Change from baseline weight. The admission (year $2005)$ in which hypertonic saline was not used (-) was compared with the admission (year 2006 or 2007) in which hypertonic saline was used (+). Units are per cent change in weight from admission to discharge. $n=18$. Data $=$ mean \pm $95 \%$ Cl. NS, $p>0.05$. sion was 247 days when HTS was not used (95\% CI 154 to 340 days) and 176 days when HTS was used (95\% CI 98 to 253 days). Figure 3 shows that HTS was associated with decrease time to next admission, but this decrease is not statistically significant $(\mathrm{p}=0.08,95 \%$ CI -9.8 to 152.7). Failure of increase in the time to next admission is probably related to short-term use of HTS in this study.

\section{CONCLUSIONS}

In this retrospective pilot study of subjects hospitalised for exacerbation of CF lung disease, use of inhaled HTS was not associated with additional benefit over standard treatment, which includes intravenous antibiotics, enhanced nutritional supplementation and airway clearance therapies. Two recently published studies showed that inhaled HTS improved outcomes in subjects with CF presumably by hydration of airway surface liquid and improved clearance of airway secretions. HTS can improve pulmonary function and mucus clearance in patients with stable CF lung disease even when used for a short time (2 weeks). ${ }^{13}{ }^{20}$ In a large, multicentre randomised controlled trial of stable subjects with $\mathrm{CF}$, twice daily inhalation of 7\% HTS over 48 weeks was shown to decrease exacerbation frequency compared with isotonic saline. ${ }^{15}$ The decrease in exacerbation frequency can also be observed in later stages of the disease. ${ }^{21}$ The only study of inpatient use of HTS to our knowledge is a study of hospitalised infants with viral bronchiolitis, where nebulised 3\% HTS was well tolerated and reduced length of stay significantly. ${ }^{22}$ In another recent study, 7\% HTS was found to be safe and well tolerated in infants with $\mathrm{CF}^{17}$

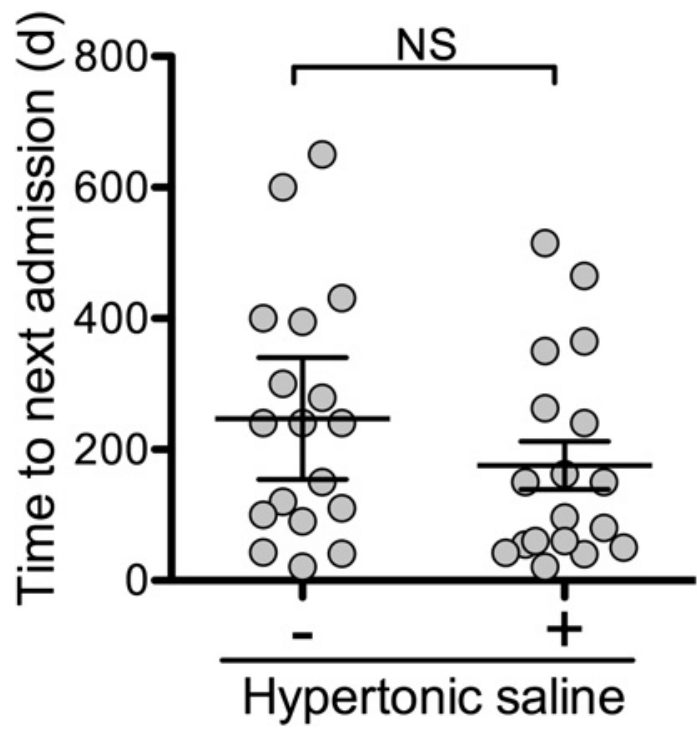

Figure 3 Time to next admission. The admission (year 2005) in which hypertonic saline was not used (-) was compared with the admission (year 2006 or 2007) in which hypertonic saline was used (+). Units are number of days between discharge and next admission with a diagnosis of acute exacerbation of cystic fibrosis lung disease. $n=18$. Data $=$ mean $\pm 95 \% \mathrm{Cl}$. NS, $\mathrm{p}>0.05$. 
Patients admitted for exacerbation of CF lung disease have been offered inhaled HTS as an adjunct to conventional therapy in our institution (and othersverbal communication). The decision to use inhaled HTS is based on the premise that the benefit will outweigh the risk of adverse events (which have been minimal in most studies) and the low cost and ease of administration of this agent. ${ }^{10-12} 14 \quad 15$ 23-26 However, inhaled HTS can induce cough ${ }^{10-12} \quad 142023 \quad 24 \quad 26$ in human subjects and has been used to provoke bronchoconstriction. $^{25} 27$ In previous studies of inhaled HTS for $\mathrm{CF}$, cough and dyspnoea have been the reasons for discontinuation of use and exclusion from study. ${ }^{14202324}$ Increased airway inflammation could cause hospitalised patients to be particularly susceptible to HTS-induced bronchospasm. One of the primary aims of this study was to examine the tolerability of HTS in this high-risk population. We did not observe an increased incidence of bronchospasm or worsening obstructive physiology when HTS was used.

A recent analysis of risk factors that are associated with failure to recover to spirometric baseline (at least $90 \%$ of the best $\mathrm{FEV}_{1}$ in the 6 months previous to an exacerbation) after intravenous antibiotic treatment reported that approximately $25 \%$ of subjects fail to recover at least 3 months after the episode. ${ }^{19}$ In our study, four out of 18 subjects failed to recover to spirometric baseline at discharge when HTS was not used, whereas two out of 16 failed to do so when HTS was used. This analysis also fails to demonstrate significant benefit of using HTS during acute exacerbation of CF, although the data from both studies are not directly comparable since patient populations with different characteristics are analysed.

Why were we unable to detect any improvement in pulmonary function tests or weight gain in this group of CF inpatients? In contrast to previous studies, ${ }^{13} 15$ this group of subjects do not present with stable lung disease. In a patient with acute exacerbation who is already receiving maximal treatment including intravenous antibiotics, chest physical therapy and rhDNase, the additional benefit of HTS could be trivial because of a ceiling effect. The fact that admission $\mathrm{FEV}_{1}$ during the visit in which HTS was used was generally higher while discharge $\mathrm{FEV}_{1}$ was similar in both visits also supports a possible ceiling effect, in which only a certain degree of improvement may be possible, even with maximal therapy.

Limitations of this study include retrospective nature of data collection from a single institution and unavailability of detailed compliance and side effect profiles. Due to its inherent retrospective nature, this study is non-randomised and does not benefit from intention-to-treat analysis. Although we examined several variables such as $\mathrm{FEV}_{1}$, sputum microbiology and co-interventions, there may be other unmeasured biases between the two admission time periods that may have diluted or masked the treatment effect. Observed power analysis reveals that our sample size and data distribution allow us to detect a difference of at least $3.6 \% \mathrm{FEV}_{1}$ (primary outcome) between admission and discharge $\mathrm{FEV}_{1}$ with $80 \%$ power (18 pairs, SD between groups of 5.44). These data will be useful for adequate power analysis prior to future related studies. The sample size is too small to allow subgroup analysis based on factors, such as baseline $\mathrm{FEV}_{1}$ and age. For the secondary outcome of weight gain (14 pairs, SD between groups of 5.65), our sample size allows detection of $4.23 \mathrm{~kg}$ in weight gain difference between admissions. In the case of time to next admission (18 pairs, SD between groups of 163.44), which was a more variable outcome, our sample size allows detection of a change of 107 days or more. The absence of increased weight gain from using HTS during hospitalisation could be the result of insufficient follow-up time, although a long-term study also failed to demonstrate a benefit. ${ }^{15}$

This study also highlights an important clinical dilemma in $\mathrm{CF}$ patient care. In the last decade, at least five new agents have shown benefits compared with placebo. $^{28}$ These valuable, placebo-controlled randomised control trials for new treatments, however, do not analyse synergy or interaction with other therapies. In real-world applications, the clinician must apply 'new' therapy in conjunction with standard treatments (eg, airway clearance techniques) and other relatively new therapies (eg, rhDNase). Questions such as 'How should the clinician prioritise these therapies?' and 'Are there important interactions between these therapies?' have been raised by those writing consensus documents for $\mathrm{CF}$ practice. ${ }^{29}$ The results of the current study demonstrate the importance of addressing these questions in wellplanned clinical trials since the outcomes of 'uncontrolled' combinations in clinical practice are unpredictable. A recently registered prospective randomised controlled trial (ANZCTR \# ACTRN12605000780651) may provide clearer answers regarding the role of inhaled HTS during hospitalisation for exacerbations of CF lung disease.

In conclusion, in this study, use of inhaled HTS during hospitalisation for acute exacerbation of CF was not associated with $\mathrm{FEV}_{1}$ improvement, additional weight gain or increased time to next admission over standard treatment in this retrospective study. The question of whether clinicians should offer or withhold this treatment to patients hospitalised for an exacerbation of CF will require a well-controlled therapeutic combination type studies showing added efficacy without added burden. As new therapies are expected, the CF research community will increasingly face the dilemma of how to combine/prioritize multiple effective treatments.

Acknowledgements The authors thank Drs Joseph Zabner and Michael Welsh for insightful discussion, Dr Bridget Zimmerman for guiding our statistical analyses and Janice Launspach and Rebecca Beek for excellent assistance.

Contributors AAP, DAS, DBH and LD contributed to the overall concept, design and interpretation of the study, data collection and manuscript preparation. All authors read and approved the final manuscript. LD guarantees the accuracy of data presented in this study. 
Funding This work was supported by the National Institutes of Health (grants K23 HL75402-5, U01 HL102288-01 and K08 Al076671).

Competing interests None.

Ethics approval Ethics approval was provided by the University of lowa Institutional Review Board (200710793).

Provenance and peer review Not commissioned; externally peer reviewed.

Data sharing statement There are no additional unpublished data in this study.

\section{REFERENCES}

1. Andersen DH. Cystic fibrosis of the pancreas and its relation to celiac disease: a clinical and pathologic study. Am J Dis Child 1938;56:344-99.

2. Rowe SM, Miller S, Sorscher EJ. Cystic fibrosis. N Engl J Med 2005;352:1992-2001.

3. Döring G, Gulbins E. Cystic fibrosis and innate immunity: how chloride channel mutations provoke lung disease. Cell Microbiol 2009;11:208-16.

4. Quinton PM. Physiological basis of cystic fibrosis: a historical perspective. Physiol Rev 1999;79:S3-22.

5. Goss $\mathrm{CH}$, Burns JL. Exacerbations in cystic fibrosis: 1. Epidemiology and pathogenesis. Thorax 2007;62:360-7.

6. Bell SC, Robinson PJ. Exacerbations in cystic fibrosis: 2. Prevention. Thorax 2007;62:723-32.

7. Smyth A, Elborn JS. Exacerbations in cystic fibrosis: 3. Management. Thorax 2008;63:180-4.

8. Chang $A B$, Bilton $D$. Exacerbations in cystic fibrosis: 4. Non-cystic fibrosis bronchiectasis. Thorax 2008;63:269-76.

9. Flume PA, Mogayzel PJ, Robinson KA, et al. Cystic fibrosis pulmonary guidelines: treatment of pulmonary exacerbations. $A m \mathrm{~J}$ Respir Crit Care Med 2009;180:802-8.

10. Suri R, Grieve R, Normand C, et al. Effects of hypertonic saline, alternate day and daily rhDNase on healthcare use, costs and outcomes in children with cystic fibrosis. Thorax 2002;57:841-6.

11. Suri R, Marshall LJ, Wallis C, et al. Effects of recombinant human DNase and hypertonic saline on airway inflammation in children with cystic fibrosis. Am J Respir Crit Care Med 2002;166:352-5.

12. Suri $R$, Metcalfe $C$, Lees $B$, et al. Comparison of hypertonic saline and alternate-day or daily recombinant human deoxyribonuclease in children with cystic fibrosis: a randomised trial. Lancet 2001;358:1316-21.

13. Donaldson SH, Bennett WD, Zeman KL, et al. Mucus clearance and lung function in cystic fibrosis with hypertonic saline. $N$ Engl J Med 2006;354:241-50.
14. Elkins MR, Bye PT. Inhaled hypertonic saline as a therapy for cystic fibrosis. Curr Opin Pulm Med 2006;12:445-52.

15. Elkins MR, Robinson M, Rose BR, et al. A controlled trial of long-term inhaled hypertonic saline in patients with cystic fibrosis. N Engl J Med 2006;354:229-40.

16. Ratjen F. Inhaled hypertonic saline produces small increases in lung function in patients with cystic fibrosis. J Pediatr 2006;149:142.

17. Subbarao P, Balkovec S, Solomon M, et al. Pilot study of safety and tolerability of inhaled hypertonic saline in infants with cystic fibrosis. Pediatr Pulmonol 2007;42:471-6.

18. Taylor LM, Kuhn RJ. Hypertonic saline treatment of cystic fibrosis. Ann Pharmacother 2007:41:481-4.

19. Sanders DB, Bittner RC, Rosenfeld M, et al. Failure to recover to baseline pulmonary function after cystic fibrosis pulmonary exacerbation. Am J Respir Crit Care Med 2010;182:627-32.

20. Eng PA, Morton J, Douglass JA, et al. Short-term efficacy of ultrasonically nebulized hypertonic saline in cystic fibrosis. Pediatr Pulmonol 1996;21:77-83.

21. Dmello D, Nayak RP, Matuschak GM. Stratified assessment of the role of inhaled hypertonic saline in reducing cystic fibrosis pulmonary exacerbations: a retrospective analysis. BMJ Open 2011;1: e000019.

22. Kuzik BA, Al-Qadhi SA, Kent S, et al. Nebulized hypertonic saline in the treatment of viral bronchiolitis in infants. $J$ Pediatr 2007;151:266-70, 270.e1.

23. Robinson M, Hemming AL, Regnis JA, et al. Effect of increasing doses of hypertonic saline on mucociliary clearance in patients with cystic fibrosis. Thorax 1997;52:900-3.

24. Robinson M, Regnis JA, Bailey DL, et al. Effect of hypertonic saline, amiloride, and cough on mucociliary clearance in patients with cystic fibrosis. Am J Respir Crit Care Med 1996;153:1503-9.

25. Schoeffel RE, Anderson SD, Altounyan RE. Bronchial hyperreactivity in response to inhalation of ultrasonically nebulised solutions of distilled water and saline. Br Med J (Clin Res Ed) 1981;283:1285-7.

26. Suri $\mathrm{R}$, Metcalfe $\mathrm{C}$, Wallis $\mathrm{C}$, et al. Predicting response to rhDNase and hypertonic saline in children with cystic fibrosis. Pediatr Pulmonol 2004;37:305-10.

27. O'Byrne PM, Gauvreau GM, Brannan JD. Provoked models of asthma: what have we learnt? Clin Exp Allergy 2009; 39:181-92.

28. Frerichs C, Smyth A. Treatment strategies for cystic fibrosis: what's in the pipeline? Expert Opin Pharmacother 2009;10:1191-202.

29. Flume PA, O'Sullivan BP, Robinson KA, et al. Cystic fibrosis pulmonary guidelines: chronic medications for maintenance of lung health. Am J Respir Crit Care Med 2007;176:957-69. 